\title{
Chest Radiography and Echocardiography Predictors of Early Hemodialysis Catheter Dysfunction
}

\author{
Kirsteen Burton ${ }^{1}$, Jeremy O’Brien ${ }^{2}$, Dheeraj Rajan ${ }^{1}$ \\ ${ }^{1}$ University of Toronto, Toronto, Canada \\ ${ }^{2}$ McGill University, Montreal, Canada \\ Email: kirsteen.burton@utoronto.ca
}

Received May 30, 2012; revised June 27, 2012; accepted July 10, 2012

\begin{abstract}
Purpose: To determine whether radiographic heart morphology and echocardiography findings were associated with early central venous catheter (CVC) failure in hemodialysis patients. Materials \& Methods: All hemodialysis catheter insertions between 1996-2007 were captured in a combined nephrology radiology database. Factors were identified that may be associated with early catheter failure. Factors examined included: echocardiography findings, radiographic evidence of flattened heart border, boot-shaped heart and left ventricular hypertrophy as well as patient comorbidities and peri-procedural characteristics. Results: Of the 132 CVC failures in 132 patients, 67 (50.8\%) occurred within 30 days of insertion. Early CVC failure was more likely to occur in patients who had insertions from the left side $\left(\chi^{2}, \mathrm{p}=0.020\right)$ and with catheter tip position outside the cavoatrial junction $\left(\chi^{2}, \mathrm{p}=0.040\right)$. CVC failure rates also differed by year of placement $\left(\chi^{2}, \mathrm{p}<0.01\right)$, if the patient had evidence of left ventricular hypertrophy $\left(\chi^{2}, \mathrm{p}=0.030\right)$, left systolic dysfunction $\left(\chi^{2}, \mathrm{p}=0.030\right)$, and hypokinetic right wall mechanics $\left(\chi^{2}, \mathrm{p}<0.01\right)$. Conclusion: Radiographic findings of left ventricular hypertrophy and echocardiography findings of left systolic dysfunction were found to be associated with early failure.
\end{abstract}

Keywords: Dialysis; Interventional Radiology; Echocardiography

\section{Introduction}

Patients with end stage kidney disease undergoing chronic hemodialysis therapy require dependable vascular access and an autogenous fistula has proven to be the most reliable conduit [1,2]. However, tunneled hemodialysis catheters continue to serve an important role in this patient population and these catheters remain especially prevalent among Canadian hemodialysis patients [3]. Although patients with CVCs have greater morbidity and mortality relative to patients with AV fistulas and grafts [4], CVC access remains beneficial in patients where AV fistulas may be impractical, where short- to intermediate-term bridge access is required until an AV fistula is developed or where vascular anatomy is unsuitable for AV fistula or graft creation [5].

Despite their limited advantages, CVCs incur low relative patency rates and significant complications, and recommendations have been made for their optimal management [6,7]. Many previous studies have identified factors that contribute to CVC failure, including infection, thrombosis and other comorbidities such as hypertension and cancer as contributory factors, however, none have examined factors related to heart anatomy [8-10]. Nu- merous studies have reported patency outcomes at 30 days $[11,12]$. And at least one case report has noted vascular access failure within 10 days, but little work has examined factors that contribute to short- versus longterm CVC failure [13].

To address the question of chest radiograph risk factors for early catheter failure, defined as failure within 30 days of insertion [14], this retrospective study included an analysis of specific heart morphologies as well as other patient and peri-procedural characteristics to identify possible risk factors for predicting hemodialysis catheter outcome.

\section{Materials and Methods}

\subsection{Patient Population}

CVC failures in hemodialysis patients were captured by a joint, regional nephrology radiology database of patients from the Toronto General Hospital during the period of 1996 to 2007. Institutional research ethics board approval was obtained for this retrospective study. CVC insertions included in the study sample were limited to new catheter insertions, inserted by interventional radiologists, with failures that occurred both within 30 and after 30 days. 
Failure was defined as failure to attain and maintain an extracorporeal blood flow sufficient $(>250 \mathrm{~mL} / \mathrm{min})$ to perform hemodialysis without significantly lengthening the hemodialysis treatment. Failures and removals attributed to renal transplantation, recovery of renal function, access creation, catheter removal by patient and sepsis were excluded. Patients with insertion via the subclavian veins, femoral hemodialysis catheters and catheter exchanges were also excluded to reduce confounding factors and focus the study on the preferred internal jugular vein access route. Given these exclusion and inclusion criteria, during the study period, 132 de novo tunneled hemodialysis catheters (inserted into 132 consecutive patients) formed the study sample.

Data collected included age, sex, comorbidities present (diabetes, hypertension, coronary artery disease (CAD), peripheral arterial vascular disease, hypercholesterolemia), presence of malignancy, therapeutic anticoagulation, type of catheter inserted, location of the tip of the catheter by posterior-anterior and lateral chest radiography (CXR) within 10 days after insertion, echocardiography results (including ventricular size, systolic/diastolic function, and ejection fraction), pre-procedure CXR heart configuration including right heart border straightening, left ventricular hypertrophy and boot-shaped heart. All hemodialysis catheters were inserted under radiological guidance with use of ultrasound to access the jugular veins and fluoroscopic guidance by board certified interventional radiologists. All catheters were cuffed with a step tip configuration with occasional split tip catheters inserted. Catheters inserted were the Uldall-Cook 16 French (Fr) (Cook Medical, Bloomington IN), Cardiomed HighFlow 14.5 Fr (CardioMed Supplies, Gormley, ON), Vaxcel 14.5 Fr (Boston Scientific, Natick, MA), Ash Split Cath (Medical Components Inc., Harleysville, PA) and Optiflow (CR Bard, Salt Lake City, UT). All catheters were tunneled from a chest incision to a neck incision and passed through a peel-away sheath via the internal jugular vein into the superior vena cava, atrial caval junction or proximal right atrium. Fibrin sheath formation was assessed at time of catheter exchange or revision. Prior to exchange, the catheter was pulled back to the venipuncture site and non-iodinated contrast was injected with digitally subtracted images obtained at two frames per second.

A total of 132 patients were included in the study sample. Demographic information and baseline risk factors of the study cohort are presented in Table 1. The mean patient age was 58.0 years (range, 18 - 88 years).

\subsection{Study Endpoints and Definitions}

Posterior-anterior and lateral chest radiographs were reviewed by three board certified radiologists for catheter tip position and radiographic appearance of the heart on chest radiographs and the consensus method was used to resolve any disagreements. Catheter tip position was determined based on location to the carina and junction of SVC with the right heart border. If the catheter tip terminated within $3 \mathrm{~cm}$ of the carina, the catheter tip was considered to be within the SVC. If the tip terminated at the junction of the SVC with the right heart border, this was considered to be the atrial caval junction. If the catheter tip extended beyond this radiographic boundary, it was considered to be within the proximal right atrium. [15,16]. Tip position was not assessed with echocardiography. Radiographic appearance of the heart was recorded as normal, right heart border straightening (where the SVC border and right heart border followed a straight line) or left ventricular hypertrophy (where the cardiothoracic ratio measured $>50 \%$ ). The primary outcome was early CVC failure, defined as a catheter failure within 30 days after the date of insertion. Comparison was made to catheters that failed beyond 30 days.

\subsection{Statistical Analysis}

Patients with early CVC failure versus those with late CVC failure were compared using the Wilcoxon ranksum test for continuous variables and the chi-square or Fisher exact tests for categorical variables. For analysis of risk factors for early failure, logistic regression analysis was used. The association of heart shape with early CVC failure was assessed using univariate logistic regression analyses. All analyses were performed using the SPSS software package, version 17.0 (SPSS Inc, Chicago, IL); $>0.05$ was considered statistically significant.

\section{Results}

One hundred and thirty-two catheters were placed in 132 patients and mean catheter failure time was 25.7 days (range, 0 - 73 days). Technical success of placement was $100 \%$. Early CVC failure (less than 30 days) occurred in 67 patients (50.8\%). The two patient groups were similar with respect to mean age and sex; however, those patients who had late CVC failure tended to have their CVCs placed in a more recent year than patients who experienced early failure. Additionally, those patients with left ventricular hypertrophy, left systolic dysfunction and hypokinetic right wall mechanics were more likely to have had early CVC failure. Furthermore, patients with early failure were more likely to have had a left-sided insertion, but a catheter tip placed in the cavo-atrial junction was less likely to result in early failure. Both patient groups had similar catheter brands, insertion sites as well as similar comorbidities (Table 1).

The causes of early catheter failure are summarized in Table 2. The greatest known cause of CVC failure 
Table 1. Patient characteristics.

\begin{tabular}{|c|c|c|c|c|c|c|c|}
\hline \multirow{2}{*}{ Variable } & \multirow{2}{*}{\multicolumn{2}{|c|}{$\begin{array}{c}\text { All insertions } \\
(\mathrm{n}=132)\end{array}$}} & \multirow{2}{*}{\multicolumn{2}{|c|}{$\begin{array}{c}\text { Early failure } \\
(\mathrm{n}=67)\end{array}$}} & \multirow{2}{*}{\multicolumn{2}{|c|}{$\begin{array}{l}\text { Late failure } \\
\qquad(\mathrm{n}=65)\end{array}$}} & \multirow{3}{*}{\begin{tabular}{|c} 
p value \\
0.88
\end{tabular}} \\
\hline & & & & & & & \\
\hline Mean age (years) & 58 & & 58 & & 58 & & \\
\hline \multicolumn{8}{|l|}{ Sex } \\
\hline Female & 67 & $50.8 \%$ & 33 & $50.8 \%$ & 32 & $49.2 \%$ & 1.00 \\
\hline Male & 65 & $49.2 \%$ & 34 & $50.7 \%$ & 33 & $49.3 \%$ & \\
\hline \multicolumn{8}{|l|}{ Side of insertion } \\
\hline Right & 84 & $63.6 \%$ & 36 & $42.9 \%$ & 48 & $57.1 \%$ & 0.02 \\
\hline Left & 48 & $36.4 \%$ & 31 & $64.6 \%$ & 17 & $35.4 \%$ & \\
\hline \multicolumn{8}{|l|}{ Catheter tip position } \\
\hline Cavo-atrial junction & 21 & 15.9 & 9 & $42.9 \%$ & 12 & $57.1 \%$ & 0.04 \\
\hline Proximal/high RA & 27 & 20.5 & 19 & $70.4 \%$ & 8 & $29.6 \%$ & \\
\hline Mid-RA & 37 & 28 & 19 & $51.4 \%$ & 18 & $48.6 \%$ & \\
\hline Low RA & 7 & $5.3 \%$ & 5 & $71.4 \%$ & 2 & $28.6 \%$ & \\
\hline RA & 25 & $18.9 \%$ & 12 & $48.0 \%$ & 13 & $52.0 \%$ & \\
\hline Not stated & 15 & $11.4 \%$ & 3 & $20.0 \%$ & 12 & $80.0 \%$ & \\
\hline \multicolumn{8}{|l|}{ Catheter brand } \\
\hline Unknown & 2 & 1.5 & 2 & $100.0 \%$ & 0 & $0.00 \%$ & 0.12 \\
\hline Uldall-Cook & 88 & $66.7 \%$ & 50 & $56.8 \%$ & 38 & $43.2 \%$ & \\
\hline Cardiomed & 18 & $13.6 \%$ & 6 & $33.3 \%$ & 12 & $66.7 \%$ & \\
\hline Ash Split & 3 & $2.3 \%$ & 1 & $33.3 \%$ & 2 & $66.7 \%$ & \\
\hline Vaxcel & 20 & $15.2 \%$ & 7 & $35.0 \%$ & 13 & $65.0 \%$ & \\
\hline Optiflow & 1 & $0.8 \%$ & 1 & $100.0 \%$ & 0 & $0.0 \%$ & \\
\hline \multicolumn{8}{|l|}{ Insertion site } \\
\hline Internal jugular & 131 & $99.2 \%$ & 66 & $50.4 \%$ & 65 & $49.6 \%$ & 0.51 \\
\hline External jugular & 1 & $0.8 \%$ & 1 & $100.0 \%$ & 0 & $0.0 \%$ & \\
\hline \multicolumn{8}{|l|}{ Year of Placement } \\
\hline 1996 & 1 & $0.8 \%$ & 0 & $0.0 \%$ & 1 & $0.8 \%$ & $<0.01$ \\
\hline 1998 & 5 & $3.8 \%$ & 3 & $60.0 \%$ & 2 & $40.0 \%$ & \\
\hline 1999 & 15 & $11.4 \%$ & 10 & $66.7 \%$ & 5 & $33.3 \%$ & \\
\hline 2000 & 18 & $13.6 \%$ & 16 & $88.9 \%$ & 2 & $11.1 \%$ & \\
\hline 2001 & 24 & $18.2 \%$ & 15 & $62.5 \%$ & 9 & $37.5 \%$ & \\
\hline 2002 & 27 & $20.5 \%$ & 13 & $48.1 \%$ & 14 & $51.9 \%$ & \\
\hline 2003 & 22 & $16.7 \%$ & 6 & $27.3 \%$ & 16 & $72.7 \%$ & \\
\hline 2004 & 12 & $9.1 \%$ & 2 & $16.7 \%$ & 10 & $83.3 \%$ & \\
\hline 2005 & 6 & $4.5 \%$ & 1 & $16.7 \%$ & 5 & $83.3 \%$ & \\
\hline 2006 & 1 & $0.8 \%$ & 1 & $100.0 \%$ & 0 & $0.0 \%$ & \\
\hline 2007 & 1 & $0.8 \%$ & 0 & $0.0 \%$ & 1 & $100.0 \%$ & \\
\hline
\end{tabular}




\section{Continued}

Hypertension

Present

114

$86.4 \%$

55

$48.2 \%$

59

$51.8 \%$

0.12

Absent

$13.6 \%$

$66.7 \%$

$33.3 \%$

Diabetes

Present

$36.4 \%$

$43.8 \%$

$56.3 \%$

0.15

Absent

$63.6 \%$

$54.8 \%$

$45.2 \%$

Coronary artery disease

Present

$59.8 \%$

$49.4 \%$

$50.6 \%$

Absent

$40.2 \%$

$52.8 \%$

$47.2 \%$

Peripheral vascular disease

Present 21

Absent

Polycystic kidney disease

Present

Absent

Prior MI

Present

Absent

Hypercholesterolemia

$\begin{array}{ll}\text { Present } & 62 \\ \text { Absent } & 70 \\ \text { Present } & \end{array}$

Present

Absent

Anticoagulated

Present

Left ventricular hypertrophy

Present

Absent

Boot-shaped heart

Present

Absent

Flattened right heart border

Present
Absent

Absent

Left ventricular cavity size

Reduced
Normal
Mildly dilated

70

59

67

65

21

111

$15.9 \%$

$84.1 \%$

$4.5 \%$

$95.5 \%$

$17.4 \%$

$82.6 \%$

62

70

$47.0 \%$

$53.0 \%$

20

$15.2 \%$

$84.8 \%$

112

$53.0 \%$

$47.0 \%$

\begin{abstract}
10
\end{abstract}
57

$47.6 \%$

$51.4 \%$

$33.3 \%$

$51.6 \%$

65

11

56

$47.8 \%$

$51.4 \%$

$51.6 \%$

$50.0 \%$

$60.0 \%$

$49.1 \%$

$55.7 \%$

$45.2 \%$

28

$74.2 \%$

$25.8 \%$

$55.3 \%$

$44.7 \%$

$50.8 \%$

$49.2 \%$

40

$30.3 \%$

$48.5 \%$

$21.2 \%$
$56.1 \%$

$35.3 \%$

$50.7 \%$

$50.8 \%$

$50.7 \%$

$50.8 \%$

$52.5 \%$

$43.8 \%$

$64.3 \%$

64

28

18
$52.4 \%$

$48.6 \%$

54

0.47

$66.7 \%$

0.33

$48.4 \%$

$52.2 \%$

0.47

$48.6 \%$

53

$48.4 \%$

0.50

$50.0 \%$

$40.0 \%$

0.26

$50.9 \%$

$44.3 \%$

0.15

$54.8 \%$

$43.9 \%$

0.03

$64.7 \%$

$49.3 \%$

0.56

$49.2 \%$

$49.3 \%$

$49.2 \%$

$32 \quad 49.2 \%$

$47.5 \%$

0.19

19
36
10

$56.3 \%$

$35.7 \%$ 


\section{Continued}

Left systolic function

Mild global dysfunction

$47.0 \%$

$43.5 \%$

$56.5 \%$

0.03

Severe global dysfunction

$40.9 \%$

$50.0 \%$

$50.0 \%$

$12.1 \%$

$81.3 \%$

$18.8 \%$

Left diastolic function

Normal

$49.2 \%$

$46.2 \%$

$53.8 \%$

0.43

Mild dysfunction

$44.7 \%$

$52.5 \%$

$47.5 \%$

Moderate dysfunction

$5.3 \%$

$71.4 \%$

$28.6 \%$

Severe dysfunction

$0.8 \%$

$100.0 \%$

$0.0 \%$

Left wall abnormalities

Normal

Septal hypertrophy

Impaired relaxation

Hypokinesia

Right ventricular cavity size

Normal

80

Enlarged

52

$60.6 \%$

$39.4 \%$

$46.2 \%$

$41.0 \%$

$56.6 \%$

$100.0 \%$

$64.7 \%$

$46.3 \%$

$57.7 \%$

$67.4 \%$

$50.6 \%$

$51.2 \%$

22

Hypokinetic

43

$32.6 \%$

85

$64.4 \%$

$35.6 \%$

40

$47.1 \%$

$57.4 \%$

$29.5 \%$

$69.0 \%$

$53.8 \%$

49

$14.4 \%$

$84.1 \%$

12

$63.2 \%$

$48.6 \%$

$50.0 \%$

$1.5 \%$

1

Table 2. Early catheter failure types.

\begin{tabular}{cccccc}
\hline & $\mathrm{n}$ & \% of total failures & Early failure (\%) & Late failure (\%) & $\mathrm{p}$ value \\
\hline Poor flow & 37 & $41.1 \%$ & $17(45.9)$ & $20(54.1)$ & 0.63 \\
Malposition & 12 & $13.3 \%$ & $5(41.7)$ & $7(58.3)$ \\
Infection & 15 & $16.7 \%$ & $9(60.0)$ & $6(40.0)$ \\
No reason given & 9 & $10.0 \%$ & $6(66.7)$ & $3(33.3)$ \\
Accessing another vein & 13 & $14.4 \%$ & $8(61.5)$ & $5(38.5)$ \\
Clot & 4 & $4.4 \%$ & $3(75.0)$ & $1(25.0)$ \\
\hline
\end{tabular}


within 30 days was poor flow (45.9\%) followed by malposition. Both patient groups had similar causes of CVC failure. There were no complications associated with catheter insertion.

\section{Discussion}

According to the United States Renal Data System (USRDS), expenditures for patients with hemodialysis catheters in 2005 were approximately $\$ 75,000$ per person per year with placement costs approaching $\$ 15,000$ [1] Given such high costs, medical professionals and manufacturers have tried to overcome perceived catheter failures and deficiencies, which include thrombosis, infection, fibrin sheath formation and occlusion [17]. Furthermore, a controversial issue that is yet to be definitively resolved is the optimal location for the catheter tip [18]. Despite multiple studies that have examined differences in patency between different catheter types and tip location [16], and despite the recommendation that plainfilm imaging should remain the standard of care for evaluation of proper CVC placement [19], little investigation has been performed regarding other predictive factors for early failure of catheters prior to insertion including cardiac anatomy as displayed by plain-film imaging and echocardiography results.

A misunderstanding of cardiovascular anatomy and the various approaches to inserting CVC's may result in increased CVC failures or complications [20]. Some studies have demonstrated that certain demographic factors or multiple percutaneous punctures per procedure have been associated with CVC complications [21]. In terms of site of introduction, multiple studies have shown that the right internal jugular vein approach is associated with lower rates of mechanical complications relative to other sites, which may be partially attributable to differences in diameter between each vein [22-24]. Within our study, we also found that catheter insertion from the right side was associated with a greater likelihood of maintained patency [25]. Beyond these results, few other factors have been examined.

The original hypothesis of our study was that the radiographic shape of the heart could predict the risk for early catheter failure. This was a personal hypothesis based on observed but unstudied outcomes. An enlarged cardiomediastinal silhouette is often associated with left ventricular hypertrophy and we hypothesized that this was associated with early catheter failure. The premise was that left ventricular hypertrophy would indicate a possible axis shift of the heart whereby the wall of the right atrium would parallel the wall of the superior vena cava (SVC). Assuming this possibility, we hypothesized that there would be a greater likelihood of the hemodialysis catheter resting against the wall of the SVC and/or right atrium leading to poor flows and early failure of the CVC [26,27]. After analysis of the data, radiographic findings of left ventricular hypertrophy was significantly associated with early failure.

In addition to radiographic findings, we examined echocardiographic findings to determine any association with early failure and found that left ventricular dysfunction was found to be significant. Left ventricular dysfunction is associated with left ventricular hypertrophy. In addition, when determining potential predictors of catheter failure, we also examined early failures versus late failures and found that there was no association between the type of catheter failure and whether failure occurred early or late.

The suggested catheter tip location by manufacturers is the right atrium/SVC junction. This position has been the subject of intense debate within recent years with the 2001 Kidney Disease Outcomes Quality Initiative (K/ DOQI) Clinical Practice Guidelines suggesting the tip be located in the SVC/right atrial junction for tunneled (cuffed) catheters and SVC/atrial junction or the SVC for non-tunneled catheters [28]. At our institution, we attempt to place the tip of the catheter in the proximal right atrium. Some have suggested that there is improved patency in this location because of the lower suggested risk of fibrin sheath formation and wall apposition [27, 29,30]. Others have suggested that right atrium positioning may be associated with an increased risk of atrial clot formation [31]. Despite our preferred location within the proximal right atrium, a number of catheters were placed with the tips terminating at the SVC or at the atrial caval junction. We observed a significantly greater risk of early catheter failure when the catheter tip was located in a position other than the cavoatrial junction and have to explanation for this observation.

Limitations of the current study include the retrospective nature of the study and the relatively small sample size. The retrospective nature of the study may have biased results, particularly in regards to the cause of failure. For instance, the absence of fibrin sheath was often inferred based on database records and is partially reflected in the $60 \%$ early failure rate for unknown causes. A prospective design may have helped determine the cause of failure in the "unknown" group. This, in turn, may have uncovered links between different heart morphologies or comorbidities and particular types of catheter failure. Furthermore, it is possible that a study with a larger sample size may better elucidate the association between left ventricular hypertrophy and increased risk of CVC failure. Also, only step tip catheters were inserted. Newer catheter shapes may influence predictive factors. Lastly, catheter tip position was determined from an on-table antero-posterior image. Catheter tip position can change significantly from a supine to upright position [18]. 
In summary, insertions from the right side were associated with maintained catheter patency whereas radiographic evidence of left ventricular hypertrophy was associated with early failure. Echocardiography findings of left systolic dysfunction was also associated with early failure.

\section{REFERENCES}

[1] B. Astor, J. Eustace, N. Powe, et al., "Type of Vascular Access and Survival among Incident Hemodialysis Patients: the Choices for Healthy Outcomes in Caring for ESRD (CHOICE) Study," Journal of the American Society of Nephrology, Vol. 16, No. 5, 2005, pp. 1449-1455. doi:10.1681/ASN.2004090748

[2] F. Quarello, G. Forneris, M. Borca, et al., "Do Central Venous Catheters Have Advantages over Arteriovenous Fistulas or Grafts?” Journal of Nephrology, Vol. 19, No. 3, 2006, pp. 265-279.

[3] D. Mendelssohn, J. Ethier, S. Elder, et al., "Haemodialysis Vascular Access Problems in Canada: Results from the Dialysis Outcomes and Practice Patterns Study (DOPPS II)," Nephrology Dialysis Transplant, Vol. 21, No. 3, 2006, pp. 721-728. doi:10.1093/ndt/gfi281

[4] D. Chand, B. Teo, R. Fatica, et al., "Influence of Vascular Access Type on Outcome Measures in Patients on Maintenance Hemodialysis," Nephron Clinical Practice, Vol. 108, No. 2, 2008, pp. 91-98. doi:10.1159/000113525

[5] A. Freeman, M. Gallagher, A. Gray-Weale, et al., "Surgical Practice to Reduce Dialysis Access Insufficiency," ANZ Journal of Surgery, Vol. 78, No. 5, 2008, pp. 377382. doi:10.1111/j.1445-2197.2008.04481.x

[6] A. Falk, "Use of the Femoral Vein as Insertion Site for Tunneled Hemodialysis Catheters," Journal of Vascular and Interventional Radiology, Vol. 18, No. 2, 2007, pp. 217-225. doi:10.1016/j.jvir.2006.12.001

[7] B. Canaud, L. Chenine, D. Henriet, et al., "Optimal Management of Central Venous Catheters for Hemodialysis," Contributions to Nephrology, Vol. 161, 2008, pp. 39-47. doi:10.1159/000129752

[8] O. Lee, J. Raque, L. Lee, et al., "Retrospective Assessment of Risk Factors to Predict Tunneled Hemodialysis Catheter Outcome," Journal of Vascular and Interventional Radiology, Vol. 15, No. 5, 2004, pp. 457-461. doi:10.1097/01.RVI.0000124942.24134.62

[9] B. Peynircioglu, F. Ozkan, M. Canyigit, et al., "Tunneled Internal Jugular Catheters in Adult Patients: Comparison of Outcomes in Hemodialysis versus Infusion Catheters," Acta Radiologica, Vol. 48, No. 6, 2007, pp. 613-619. doi:10.1080/02841850701342104

[10] T. Wilkin, M. Kraus, K. Lane, et al., "Internal Jugular Vein Thrombosis Associated with Hemodialysis Catheters,” Radiology, Vol. 228, No. 3, 2003, pp. 697-700. doi:10.1148/radiol.2283020681

[11] A. Bakken, C. Protack, W. Saad, et al., "Long-Term Outcomes of Primary Angioplasty and Primary Stenting of Central Venous Stenosis in Hemodialysis Patients," Journal of Vascular Surgery, Vol. 45, No. 4, 2007, pp. 776-
783. doi:10.1016/j.jvs.2006.12.046

[12] A. Falk and S. Parthasarathy, "Conversion of Temporary Hemodialysis Catheters to Tunneled Hemodialysis Catheters,” Clinical Nephrology, Vol. 63, No. 3, 2005, pp. 209214.

[13] M. Napoli, A. Montinaro, F. Russo, et al., "Ultrasound Guided Brachial Arterial Angioplasty during the Creation of a Radio-Cephalic Arteriovenous Fistula: A Case Report,” Journal of Vascular Access, Vol. 7, No. 1, 2006, pp. 38-42.

[14] J. E. Silberzweig, D. Sacks, A. S. Khorsandi, et al., "Reporting Standards for Central Venous Access," Journal of Vascular and Interventional Radiology, Vol. 14, No. 9, 2003, pp. 443-452. doi:10.1097/01.RVI.0000094617.61428.bc

[15] M. Mahlon and H. Yoon, "CT Angiography of the Superior Vena Cava: Normative Values and Implications for Central Venous Catheter Position,” Journal of Vascular and Interventional Radiology, Vol. 18, No. 9, 2007, pp. 1106-1110. doi:10.1016/j.jvir.2007.06.002

[16] K. Albrecht, H. Nave, D. Breitmeier, et al., “Applied Anatomy of the Superior Vena Cava-The Carina as a Landmark to Guide Central Venous Catheter Placement," British Journal of Anaesthesia, Vol. 92, No. 1, 2004, pp. 75-77. doi:10.1093/bja/aeh013

[17] L. Oguzkurt, F. Tercan, D. Torun, et al., "Impact of Short-Term Hemodialysis Catheters on the Central Veins: A Catheter Venographic Study," European Journal of Radiology, Vol. 52, No. 3, 2004, pp. 293-299. doi:10.1016/j.ejrad.2003.12.004

[18] T. Vesely, "Central Venous Catheter Tip Position: A Continuing Controversy,” Journal of Vascular and Interventional Radiology, Vol. 14, No. 5, 2003, pp. 527-534. doi:10.1097/01.RVI.0000071097.76348.72

[19] G. Abood, K. Davis, T. Esposito, et al., "Comparison of Routine Chest Radiograph versus Clinician Judgment to Determine Adequate Central Line Placement in Critically Ill Patients,” Journal of Trauma, Vol. 63, No. 1, 2007, pp. 50-56. doi:10.1097/TA.0b013e31806bf1a3

[20] J. Boon, A. van Schoor, P. Abrahams, et al., "Central Venous Catheterization-An Anatomical Review of a Clinical Skill-Part 1: Subclavian Vein via the Infraclavicular Approach,” Clinical Anatomy, Vol. 20, No. 6, 2007, pp. 602-611. doi:10.1002/ca.20486

[21] L. Eisen, M. Narasimhan, J. Berger, et al., "Mechanical Complications of Central Venous Catheters," Journal of Intensive Care Medicine, Vol. 21, No. 1, 2006, pp. 40-46. doi:10.1177/0885066605280884

[22] R. Botha, A. van Schoor, J. Boon, et al., "Anatomical Considerations of the Anterior Approach for Central Venous Catheter Placement," Clinical Anatomy, Vol. 19, No. 2, 2006, pp. 101-105. doi:10.1002/ca.20240

[23] A. Pikwer, L. Baath, B. Davidson, et al., "The incidence and Risk of Central Venous Catheter Malpositioning: A Prospective Cohort Study in 1619 Patients,” Anaesthesia and Intensive Care, Vol. 36, No. 1, 2008, pp. 30-37.

[24] H. Hamilton and D. Foxcroft, "Central Venous Access Sites for the Prevention of Venous Thrombosis, Stenosis 
and Infection in Patients Requiring Long-Term Intravenous Therapy," Cochrane Database Systematic Review, Vol. 3, 2007.

[25] C. Male, P. Chait, M. Andrew, et al., "Central Venous Line-Related Thrombosis in Children: Association with Central Venous Line Location and Insertion Technique," Blood, Vol. 101, No. 11, 2003, pp. 4273-4278. doi:10.1182/blood-2002-09-2731

[26] D. Xiang, E. Verbeken, A. Van Lommel, et al., "Composition and Formation of the Sleeve Enveloping a Central Venous Catheter," Journal of Vascular Surgery, Vol. 28, No. 2, 1998, pp. 260-271. doi:10.1016/S0741-5214(98)70162-4

[27] S. Ford and P. Manley, "Indwelling Cardiac Catheters: An Autopsy Study of Associated Endocardial Lesions," Archives of Pathology and Laboratory Medicine, Vol. 106, No. 7, 1982, pp. 314-317.
[28] N. K. Foundation, “K/DOQI Clinical Practice Guidelines for Vascular Access," American Journal of Kidney Disease, Vol. 37, No. 1, 2001, pp. S137-S181. doi:10.1016/S0272-6386(01)70007-8

[29] V. Puel, M. Caudry and P. Le Metayer, “Tunneled Catheter Associated Atrial Thrombi: Successful Treatment with Chronic Anticoagulation," Hemodialysis International, Vol. 5, 1993, pp. 32-36.

[30] A. Cadman, J. A. Lawrance, L. Fitzsimmons, et al., "To Clot or Not to Clot? That is the Question in Central Venous Catheters," Clinical Radiology, Vol. 59, No. 4, 2004, pp. 349-355.

[31] E. J. Kingdon, S. G. Holt, J. Davar, et al., "Atrial Thrombus and Central Venous Dialysis Catheters," American Journal of Kidney Diseases, Vol. 38, No. 3, 2001, pp. 631-639. 